The online version of this article is published within an Open Access environment subject to the conditions of the Creative Commons Attribution-NonCommercial-ShareAlike licence $<$ http://creativecommons.org/licenses/by-nc-sa/3.0/>. The written permission of Cambridge University Press must be obtained for commercial re-use.

doi:10.1017/jfm.2013.193

\title{
Two-dimensional magnetohydrodynamic turbulence in the limits of infinite and vanishing magnetic Prandtl number
}

\author{
Chuong V. Tran $\dagger$, Xinwei Yu and Luke A. K. Blackbourn \\ School of Mathematics and Statistics, University of St Andrews, St Andrews KY16 9SS, UK \\ Department of Mathematical and Statistical Sciences, University of Alberta, Edmonton, Alberta T6G 2G1, \\ Canada
}

(Received 29 October 2012; revised 26 February 2013; accepted 9 April 2013; first published online 14 May 2013)

We study both theoretically and numerically two-dimensional magnetohydrodynamic turbulence at infinite and zero magnetic Prandtl number $P m$ (and the limits thereof), with an emphasis on solution regularity. For $P m=0$, both $\|\omega\|^{2}$ and $\|j\|^{2}$, where $\omega$ and $j$ are, respectively, the vorticity and current, are uniformly bounded. Furthermore, $\|\nabla j\|^{2}$ is integrable over $[0, \infty)$. The uniform boundedness of $\|\omega\|^{2}$ implies that in the presence of vanishingly small viscosity $v$ (i.e. in the limit $P m \rightarrow 0$ ), the kinetic energy dissipation rate $v\|\omega\|^{2}$ vanishes for all times $t$, including $t=\infty$. Furthermore, for sufficiently small $P m$, this rate decreases linearly with $P m$. This linear behaviour of $v\|\omega\|^{2}$ is investigated and confirmed by high-resolution simulations with $\mathrm{Pm}$ in the range $[1 / 64,1]$. Several criteria for solution regularity are established and numerically tested. As $P m$ is decreased from unity, the ratio $\|\omega\|_{\infty} /\|\omega\|$ is observed to increase relatively slowly. This, together with the integrability of $\|\nabla j\|^{2}$, suggests global regularity for $P m=0$. When $P m=\infty$, global regularity is secured when either $\|\nabla \boldsymbol{u}\|_{\infty} /\|\omega\|$, where $\boldsymbol{u}$ is the fluid velocity, or $\|j\|_{\infty} /\|j\|$ is bounded. The former is plausible given the presence of viscous effects for this case. Numerical results over the range $P m \in[1,64]$ show that $\|\nabla \boldsymbol{u}\|_{\infty} /\|\omega\|$ varies slightly (with similar behaviour for $\left.\|j\|_{\infty} /\|j\|\right)$, thereby lending strong support for the possibility $\|\nabla \boldsymbol{u}\|_{\infty} /\|\omega\|<\infty$ in the limit $P m \rightarrow \infty$. The peak of the magnetic energy dissipation rate $\mu\|j\|^{2}$ is observed to decrease rapidly as $P m$ is increased. This result suggests the possibility $\|j\|^{2}<\infty$ in the limit $P m \rightarrow \infty$. We discuss further evidence for the boundedness of the ratios $\|\omega\|_{\infty} /\|\omega\|,\|\nabla \boldsymbol{u}\|_{\infty} /\|\omega\|$ and $\|j\|_{\infty} /\|j\|$ in conjunction with observation on the density of filamentary structures in the vorticity, velocity gradient and current fields.

Key words: MHD and electrohydrodynamics, MHD turbulence, turbulence simulation

\section{Introduction}

The possible regularization of fluid motion in three dimensions by viscosity is a fundamental mathematical problem of both theoretical and practical interest. The 
significance of this notoriously difficult problem can be appreciated by the fact that the classical theory of turbulence is formulated on the hypothesis of non-zero energy dissipation in the inviscid limit. This hypothesis implicitly assumes finite-time singularities in the inviscid case, because otherwise the energy dissipation rate would not tend to a non-zero limit (in a finite time) in the presence of vanishingly small viscosity. Presumably, viscous effects regularize the dynamics, giving rise to a positive limiting energy dissipation rate. Thus far, this deceptively simple picture has eluded mathematical verification. For two-dimensional (2D) fluids, the problem of regularity and limiting dissipation is relatively more tractable in certain cases, yet remains highly challenging in others. On the one hand, by virtue of vorticity conservation, no finite-time singularities can develop in the 2D Euler dynamics. By the same token, 2D geophysical fluid models, which conserve the potential vorticity, are globally regular. As a result, in the presence of viscosity and for $t<\infty$, the enstrophy and potential enstrophy become conserved in the inviscid limit. For a semi-analytic proof of enstrophy conservation without the constraint $t<\infty$, see Tran \& Dritschel (2006). Some numerical demonstration of this fact can be found in Dritschel, Tran \& Scott (2007). On the other hand, it is well-known that the 2D surface quasi-geostrophic and magnetohydrodynamic (MHD) equations with usual molecular diffusion (or weaker dissipation mechanisms, see the discussion below and the theorem in $\S 2$ for the MHD case and Kiselev, Nazarov \& Volberg (2007) for the surface quasi-geostrophic case) possess globally regular solutions. However, the issue of regularity of the ideal dynamics remains outstanding. Similarly, the problem of limiting energy dissipation has not been fully understood (cf. Tran, Blackbourn \& Scott 2011; Blackbourn \& Tran 2012).

Recent mathematical studies of the 2D MHD equations have derived several regularity criteria concerning the strength of the generalized diffusion operators $-(-\Delta)^{\alpha}$, for $\alpha \geqslant 0$, which replace the usual molecular diffusion operator $\Delta$ (Wu 2011; Tran, Yu \& Zhai 2013a). Since the induction equation is linear in the magnetic field, global regularity is possible in the absence of magnetic diffusion, provided that a strong enough dissipation mechanism is applied to the velocity field, so as to keep the velocity gradients bounded (this is true for any number of dimensions, see Tran, Yu \& Zhai (2013b) and references therein). On the other hand, since vortex stretching is absent in two dimensions, global regularity is possible in the absence of momentum diffusion, provided that a sufficiently strong dissipation mechanism is applied to the magnetic field, so as to suppress magnetic stretching and keep the current gradients (which appear in the vorticity equation) bounded. Indeed, Tran et al. (2013a) have proven global regularity for either hyperviscosity (represented by $\left.(-\Delta)^{\alpha}\right)$ alone with $\alpha \geqslant 2$ or magnetic hyperdiffusion alone with $\alpha>2$. As far as the classical energy method is concerned, these bounds for $\alpha$ appear optimal. However, physical arguments and numerical analysis indicate otherwise. More precisely, Blackbourn \& Tran (2012) suggest that for fully developed turbulence from a large-scale energy reservoir, the conversion between kinetic and magnetic energy becomes quasi-steady (dynamo saturation in the three-dimensional context). This may correspond to a state of complete or nearly complete depletion of nonlinearities (cf. Tobias \& Cattaneo (2008) and the concluding remarks of Blackbourn \& Tran (2012)). Hence, excessively strong dissipation mechanisms would be unnecessary to prevent finite-time singularities.

In this study, we investigate the possibility of solution regularity of the 2D MHD equations with usual molecular diffusion when either one of the diffusion channels is removed, i.e. when either $P m=0$ or $P m=\infty$. Here $P m=\nu / \mu$ is the magnetic Prandtl number, where $v$ and $\mu$ are the viscosity and magnetic diffusivity, 
respectively. Regularity in these cases would correspond to drastic improvements to the mathematical results discussed in the preceding paragraph (reducing the upper bounds for $\alpha$ from two to one). For $P m=0$, both $\|\omega\|^{2}$ and $\|j\|^{2}$ are uniformly bounded and $\|\nabla j\|^{2}$ is integrable over $[0, \infty)$. Here $\omega$ and $j$ are, respectively, the vorticity and current and $\|\cdot\|$ denotes the usual $L^{2}$ norm. The uniform boundedness of $\|\omega\|^{2}$ implies that in the presence of vanishingly small viscosity (i.e. in the limit $P m \rightarrow 0$ ), the kinetic energy dissipation rate $v\|\omega\|^{2}$ vanishes for all times $t$, including $t=\infty$. Furthermore, for sufficiently small $\mathrm{Pm}$, this rate decreases linearly with $\mathrm{Pm}$. Several criteria for solution regularity are derived and numerically tested. The results from high-resolution simulations over the range $P m \in[1 / 64,64]$ show a mild increase of the ratio $\|\omega\|_{\infty} /\|\omega\|$, where $\|\omega\|_{\infty}$ is the vorticity supremum, as $P m$ is decreased from unity. This provides strong support for the possibility $\|\omega\|_{\infty} /\|\omega\|<\infty$ in the limit $P m \rightarrow 0$ and, together with the integrability of $\|\nabla j\|^{2}$, suggests global regularity for $P m=0$. On the other hand, when $P m$ is increased from unity, both the ratios $\|\nabla \boldsymbol{u}\|_{\infty} /\|\omega\|$, where $\boldsymbol{u}$ is the fluid velocity, and $\|j\|_{\infty} /\|j\|$ are observed to increase slowly, thereby favouring the possibility $\|\nabla \boldsymbol{u}\|_{\infty} /\|\omega\|<\infty$ and $\|j\|_{\infty} /\|j\|<\infty$ in the limit $P m \rightarrow \infty$. The observed behaviour of either ratio implies uniform boundedness of $\|j\|$, and hence vanishing magnetic energy dissipation in the presence of vanishing resistivity. In either case, global regularity immediately follows. Evidence for the boundedness of $\|\omega\|_{\infty} /\|\omega\|,\|\nabla \boldsymbol{u}\|_{\infty} /\|\omega\|$ and $\|j\|_{\infty} /\|j\|$ is discussed in conjunction with further numerical observations on the filamentary structures of the vorticity, velocity gradient and current fields.

This paper is organized as follows. Section 2 provides a brief review of some wellknown mathematical results and recent findings, which constitute the framework for the present study. Section 3 features some theoretical considerations of the dynamical behaviour in the limits $P m \rightarrow 0$ and $P m \rightarrow \infty$ and of the possibility of solution regularity for $P m=0$ and $P m=\infty$. The main results of this study are presented in $\S 4$, including numerical confirmation of and evidence for the theoretical results of $\S 3$. Concluding remarks are given in $\S 5$.

\section{Background}

The MHD equations governing the motion and evolution of the internally generated magnetic field of an electrically conducting and incompressible fluid under no influence from external magnetic fields or mechanical forces are

$$
\begin{gathered}
\frac{\partial \boldsymbol{u}}{\partial t}+(\boldsymbol{u} \cdot \nabla) \boldsymbol{u}+\nabla p=(\boldsymbol{b} \cdot \nabla) \boldsymbol{b}+v \Delta \boldsymbol{u}, \\
\frac{\partial \boldsymbol{b}}{\partial t}+(\boldsymbol{u} \cdot \nabla) \boldsymbol{b}=(\boldsymbol{b} \cdot \nabla) \boldsymbol{u}+\mu \Delta \boldsymbol{b}, \\
\nabla \cdot \boldsymbol{u}=0=\nabla \cdot \boldsymbol{b},
\end{gathered}
$$

where all notation is standard. In the momentum equation $(2.1)$, the term $(\boldsymbol{b} \cdot \nabla) \boldsymbol{b}$ represents the Lorentz force. In the induction equation (2.2), the stretching term $(\boldsymbol{b} \cdot \nabla) \boldsymbol{u}$ allows the magnetic induction $\boldsymbol{b}$ to be amplified by the fluid velocity gradients. This amplification (known as dynamo action in the three-dimensional context) necessarily results in an increase of the magnetic energy at the expense of kinetic energy as the total energy is conserved in ideal dynamics. Given a limited kinetic energy reservoir, dynamo action inevitably weakens the velocity field to some extent and necessarily becomes saturated. In three dimensions, this may result in a reduction of energy transfer to small scales (i.e. some suppression of turbulence). 
Indeed, Iroshnikov (1964) and Kraichnan (1965) suggested such a reduction on the basis of Alfvén wave effects (see also Moffatt 1967). See, however, the competing theory of Sridhar \& Goldreich (1994) and Goldreich \& Sridhar (1995). There exist ample numerical results in support of both theories (Verma et al. 1996; Galtier, Pouquet \& Mangeney 2005; Ng et al. 2010; Beresnyak 2011). It is interesting to note that in the Rayleigh-Taylor instability problem, the growth of a disturbance (to the interface of two conducting fluids of different densities with the heavier on top) can be suppressed by a magnetic field (Chambers \& Forbes 2011).

Two-dimensional dynamics lack the underpinning direct energy transfer to make sense of the Kraichnan-Iroshnikov theory (Blackbourn \& Tran 2012). In the absence of vortex stretching, the energy conversion via the Lorentz force (and magnetic stretching) is responsible for the direct energy transfer. This force breaks the vorticity conservation of the ideal dynamics, which would otherwise be effectively linear, as far as the small scales are concerned. It has long been recognized that the action of this force on the vorticity is relatively weak (Pouquet 1978; Orszag \& Tang 1979), presumably owing to some correlation between the vorticity and current fields. Hence, the degree of nonlinearity of 2D MHD turbulence can be accordingly low. Recently, Blackbourn \& Tran (2012) raised the possibility of complete or nearly complete depletion of nonlinearity for fully developed turbulence, upon which the conversion between the kinetic and magnetic energy becomes quasi-steady. A complete depletion of nonlinearity would mean that the term $\nabla \times(\boldsymbol{b} \cdot \nabla) \boldsymbol{b}$, which amplifies the vorticity, is not significantly greater than the vorticity. Note that quasi-steady energy conversion means that the energy conversion term $\boldsymbol{u} \cdot(\boldsymbol{b} \cdot \nabla) \boldsymbol{b}$ on average fluctuates about zero.

Throughout this study (except for Appendix), absolute constants (usually of order unity) are denoted by $c$ while dimensional constants, which may or may not depend on physical parameters, are denoted by $C$. These constants may vary from one line to another. As in the introductory section, the respective notation $\|\cdot\|$ and $\|\cdot\|_{\infty}$ is used for $L^{2}$ and $L^{\infty}$ norms. Other $L^{p}$ norms are explicitly specified by the subscript $L^{p}$. Bounded-mean-oscillation (BMO) norms are identified with the subscript BMO. For a brief review of BMO spaces and some of their basic properties used in this study, see Appendix.

Consider a solution evolving from a smooth initial condition $\{\boldsymbol{u}, \boldsymbol{b}\}=\left\{\boldsymbol{u}_{0}, \boldsymbol{b}_{0}\right\}$ and some time $T>0$. The CKS criterion (Caflisch, Klapper \& Steel 1997), which has become the main tool in proving regularity of the MHD equations with or without dissipation, states that the solution remains smooth at least shortly beyond $t=T$ if

$$
\int_{0}^{T}\left(\|\omega\|_{\infty}+\|\boldsymbol{j}\|_{\infty}\right) \mathrm{d} t<\infty,
$$

where $\boldsymbol{\omega}=\boldsymbol{\nabla} \times \boldsymbol{u}$ and $\boldsymbol{j}=\boldsymbol{\nabla} \times \boldsymbol{b}$ are the vorticity and current, respectively. This criterion is a straightforward extension of the celebrated BKM criterion (Beale, Kato \& Majda 1984) for usual (non-magnetic) fluids governed by the Euler or Navier-Stokes equations. As in the BKM criterion, where the BMO norm of the vorticity $\|\omega\|_{B M O}$ can replace $\|\omega\|_{\infty}$ (Kozono \& Tanuichi 2000), we have the following slightly weaker version of (2.4):

$$
\int_{0}^{T}\left(\|\boldsymbol{\omega}\|_{B M O}+\|\boldsymbol{j}\|_{B M O}\right) \mathrm{d} t<\infty .
$$

Indeed, (2.5) is weaker than (2.4) because for any function $f$, one has $\|f\|_{B M O} \leqslant 2\|f\|_{\infty}$ (see Appendix). 
The 2D MHD system possesses globally smooth solutions when both $v$ and $\mu$ are positive. It turns out that dissipation mechanisms much weaker than the usual molecular diffusion of both the velocity and magnetic fields are capable of regularizing the dynamics. Furthermore, global regularity can be secured with partial hyperdissipation. These facts are described in detail in the following theorem.

THEOREM 2.1 (Tran et al. 2013a). Consider the 2D MHD equations

$$
\begin{gathered}
\frac{\partial \boldsymbol{u}}{\partial t}+(\boldsymbol{u} \cdot \nabla) \boldsymbol{u}+\nabla p=(\boldsymbol{b} \cdot \nabla) \boldsymbol{b}-v(-\Delta)^{\alpha} \boldsymbol{u}, \\
\frac{\partial \boldsymbol{b}}{\partial t}+(\boldsymbol{u} \cdot \nabla) \boldsymbol{b}=(\boldsymbol{b} \cdot \nabla) \boldsymbol{u}-\mu(-\Delta)^{\beta} \boldsymbol{b}, \\
\nabla \cdot \boldsymbol{u}=0=\nabla \cdot \boldsymbol{b},
\end{gathered}
$$

where the viscous and magnetic diffusion terms are replaced by $-v(-\Delta)^{\alpha} \boldsymbol{u}$ and $-\mu(-\Delta)^{\beta} \boldsymbol{b}$, respectively. Given $\left\{\boldsymbol{u}_{0}, \boldsymbol{b}_{0}\right\}$ as smooth as necessary (essentially twice differentiable), the system is globally regular for any one of the following conditions.

$$
\begin{array}{r}
\text { (i) } \alpha \geqslant 1 / 2 \text { and } \beta \geqslant 1, \quad \text { (ii) } 0<\alpha<1 / 2 \text { and } 2 \alpha+\beta>2, \\
\text { (iii) } v=0 \text { and } \beta>2, \quad \text { (iv) } \alpha \geqslant 2 \text { and } \mu=0 .
\end{array}
$$

As far as the classical energy method is concerned, the results in the above theorem, together with global regularity in the case $\alpha \geqslant 1, \alpha+\beta \geqslant 2$ proved by Wu (2011), appear optimal. In this study, we are interested in (iii) and (iv), where theoretical and numerical evidence suggests a dramatic improvement, essentially reducing the lower bounds for $\alpha$ and $\beta$ to unity.

\section{Theoretical considerations}

This section considers some theoretical aspects of 2D MHD turbulence, particularly the plausibility of global regularity when either $P m=0$ or $P m=\infty$. The former case is of practical interest as plasmas in nature and liquid metals in laboratories are relatively low in viscosity but high in resistivity. In fact, $\mathrm{Pm}$ can be as low as $10^{-5}-10^{-3}$ in stellar interiors and $10^{-5}$ in liquid metals (Iskakov et al. 2007; Dritschel \& Tobias 2012).

\subsection{The limit $P m \rightarrow 0$}

By multiplying (2.1) and (2.2) by $\boldsymbol{u}$ and $\boldsymbol{b}$, respectively, summing up and integrating the resulting equation, we obtain (by virtue of energy conservation of the ideal dynamics)

$$
\frac{1}{2} \frac{\mathrm{d}}{\mathrm{d} t}\left(\|\boldsymbol{u}\|^{2}+\|\boldsymbol{b}\|^{2}\right)=-v\|\omega\|^{2}-\mu\|j\|^{2} \leqslant-\mu\|j\|^{2} .
$$

Here $\omega$ and $j$ are the (scalar) vorticity and current, respectively. It follows that

$$
\int_{0}^{t}\|j\|^{2} \mathrm{~d} \tau \leqslant \frac{1}{2 \mu}\left(\left\|\boldsymbol{u}_{0}\right\|^{2}+\left\|\boldsymbol{b}_{0}\right\|^{2}\right),
$$

for all $t$, including $t=\infty$, for which the inequality becomes an equality if $v=0$.

The governing equations for $\omega$ and $j$ are given by

$$
\frac{\partial \omega}{\partial t}+\boldsymbol{u} \cdot \nabla \omega=\boldsymbol{b} \cdot \nabla j+v \Delta \omega,
$$




$$
\frac{\partial j}{\partial t}+\boldsymbol{u} \cdot \nabla j=\boldsymbol{b} \cdot \nabla \omega+B(\nabla \boldsymbol{u}, \nabla \boldsymbol{b})+\mu \Delta j .
$$

In (3.4), B( $\nabla \boldsymbol{u}, \nabla \boldsymbol{b})$ is bilinear in $\nabla \boldsymbol{u}$ and $\nabla \boldsymbol{b}$ and given by

$$
B(\nabla \boldsymbol{u}, \nabla \boldsymbol{b})=2 \frac{\partial b_{1}}{\partial x}\left(\frac{\partial u_{2}}{\partial x}+\frac{\partial u_{1}}{\partial y}\right)+2 \frac{\partial u_{2}}{\partial y}\left(\frac{\partial b_{2}}{\partial x}+\frac{\partial b_{1}}{\partial y}\right),
$$

where $\boldsymbol{u}=\left(u_{1}, u_{2}\right)$ and $\boldsymbol{b}=\left(b_{1}, b_{2}\right)$. By multiplying (3.3) and (3.4) by $\omega$ and $j$, respectively, summing up and integrating the resulting equation, we obtain

$$
\frac{1}{2} \frac{\mathrm{d}}{\mathrm{d} t}\left(\|\omega\|^{2}+\|j\|^{2}\right)=\int j B(\nabla \boldsymbol{u}, \nabla \boldsymbol{b}) \mathrm{d} \boldsymbol{x}-v\|\nabla \omega\|^{2}-\mu\|\nabla j\|^{2},
$$

where only the triple-product term involving $B$ survives (the two triple-product terms involving $\boldsymbol{u}$ vanish while the two triple-product terms involving $\boldsymbol{b}$ cancel each other, thanks to the divergence-free properties $\boldsymbol{\nabla} \cdot \boldsymbol{u}=\boldsymbol{\nabla} \cdot \boldsymbol{b}=0$ ). This term can be estimated as follows:

$$
\begin{aligned}
\int j B(\nabla \boldsymbol{u}, \nabla \boldsymbol{b}) \mathrm{d} \boldsymbol{x} & \leqslant\|\nabla \boldsymbol{u}\|\|j\|_{L^{4}}\|\nabla \boldsymbol{b}\|_{L^{4}} \leqslant c\|\omega\|\|j\|_{L^{4}}^{2} \\
& \leqslant c\|\omega\|\|j\|\|\nabla j\| \leqslant \frac{c}{2 \mu}\|\omega\|^{2}\|j\|^{2}+\frac{\mu}{2}\|\nabla j\|^{2} .
\end{aligned}
$$

Here the first inequality is due to Hölder's inequality and the second inequality follows from the relation $\|\nabla \boldsymbol{b}\|_{L^{4}} \leqslant c\|j\|_{L^{4}}$, which is a consequence of the boundedness of Riesz operators on $L^{p}$ spaces $(1<p<\infty)$, see e.g. Stein (1970). The Gagliardo-Nirenberg inequality $\|j\|_{L^{4}}^{2} \leqslant c\|j\|\|\nabla j\|$ (see e.g. Nirenberg (1959) for a complete discussion of such a class of inequalities) and Young's inequality have been used in the subsequent steps. Substituting (3.7) into (3.6) yields

$$
\frac{\mathrm{d}}{\mathrm{d} t}\left(\|\omega\|^{2}+\|j\|^{2}\right)+\mu\|\nabla j\|^{2} \leqslant \frac{c}{\mu}\|\omega\|^{2}\|j\|^{2} \leqslant \frac{c}{\mu}\left(\|\omega\|^{2}+\|j\|^{2}\right)\|j\|^{2} .
$$

By applying Gronwall's lemma to (3.8) (or directly integrating (3.8) with the integrating factor $\left.\exp \left\{-c \int_{0}^{t}\|j\|^{2} \mathrm{~d} \tau / \mu\right\}\right)$ we obtain

$$
\begin{aligned}
\|\omega\|^{2}+\|j\|^{2}+\mu \int_{0}^{t}\|\nabla j\|^{2} \mathrm{~d} \tau & \leqslant\left(\left\|\omega_{0}\right\|^{2}+\left\|j_{0}\right\|^{2}\right) \exp \left\{\frac{c}{\mu} \int_{0}^{t}\|j\|^{2} \mathrm{~d} \tau\right\} \\
& \leqslant\left(\left\|\omega_{0}\right\|^{2}+\left\|j_{0}\right\|^{2}\right) \exp \left\{\frac{c}{2 \mu^{2}}\left(\left\|\boldsymbol{u}_{0}\right\|^{2}+\left\|\boldsymbol{b}_{0}\right\|^{2}\right)\right\},
\end{aligned}
$$

where $\omega_{0}=\omega(x, y, 0)$ and $j_{0}=j(x, y, 0)$.

Given $\mu>0$, two important results can be readily deduced from (3.9). First, both $\|\omega\|$ and $\|j\|$ are bounded independently of viscosity for $t>0$. This result was known to Lei \& Zhou (2009) and Cao \& Wu (2011). The implication is that in the presence of vanishingly small viscosity, the kinetic energy dissipation rate $v\|\omega\|^{2}$ tends to zero. Furthermore, for sufficiently small $P m$, the approach $v\|\omega\|^{2} \rightarrow 0$ becomes linear in $v$, approximately given by $\approx \mathcal{v} \Omega^{2}$, where $\Omega$ is an upper bound for $\|\omega\|$. The regime of this linear behaviour turns out to be fully accessible to numerical simulations. Indeed, the results reported in $\S 4$ show a nearly linear decrease of $v\|\omega\|^{2}$ with $v$ even for moderately small Pm. Second, we have

$$
\int_{0}^{t}\|\nabla j\|^{2} \mathrm{~d} \tau<\infty
$$


Since in two dimensions, $\|j\|_{B M O}$ can be bounded from above in terms of $\|\nabla j\|$, namely $\|j\|_{B M O} \leqslant c\|\nabla j\|$ (see Appendix), one has

$$
\int_{0}^{t}\|j\|_{B M O} \mathrm{~d} \tau<\infty
$$

which is half of the improved CKS regularity criterion (2.5).

The other half of the CKS criterion concerning $\|\omega\|_{B M O}$ is not available although $\|\omega\|$ is uniformly bounded. This half would immediately follow if $\|\omega\|_{B M O} /\|\omega\|<\infty$, and global regularity would be secured. In $\S 4$ the results from numerical simulations indicate a mild growth of the ratio $\|\omega\|_{\infty} /\|\omega\|$ for a range of small Pm, thereby suggesting global regularity. Note that for solutions with $\|\omega\|_{B M O} /\|\omega\| \leqslant F(t)$, regularity beyond $t=T$ is guaranteed once $\int_{0}^{T} F(t) \mathrm{d} t$ is bounded, as can be seen from the fact that

$$
\int_{0}^{T}\|\omega\|_{B M O} \mathrm{~d} t \leqslant \int_{0}^{T}\|\omega\| F(t) \mathrm{d} t \leqslant \Omega \int_{0}^{T} F(t) \mathrm{d} t<\infty .
$$

Here, again, $\Omega$ is an upper bound for $\|\omega\|$. In particular, for regularity beyond $t=T$, it is sufficient to require that

$$
\frac{\|\omega\|_{B M O}}{\|\omega\|} \leqslant C(T-t)^{-\alpha}
$$

for $\alpha<1$.

\subsection{The limit $P m \rightarrow \infty$}

For fixed $v>0$, the parallel of (3.2) is

$$
\int_{0}^{t}\|\omega\|^{2} \mathrm{~d} \tau \leqslant \frac{1}{2 v}\left(\left\|\boldsymbol{u}_{0}\right\|^{2}+\left\|\boldsymbol{b}_{0}\right\|^{2}\right),
$$

for all $t$, including $t=\infty$, for which the inequality becomes an equality if $\mu=0$. Now recall (3.6). To take advantage of (3.14), we consider two distinct estimates (both different from the one above) for the triple-product term. First, we have

$$
\begin{aligned}
\int j B(\nabla \boldsymbol{u}, \nabla \boldsymbol{b}) \mathrm{d} \boldsymbol{x} & \leqslant\|j\|_{\infty}\|\nabla \boldsymbol{u}\|\|\nabla \boldsymbol{b}\|=\|j\|_{\infty}\|\omega\|\|j\| \\
& \leqslant \frac{\|j\|_{\infty}}{\|j\|}\|\omega\|\left(\|\omega\|^{2}+\|j\|^{2}\right)
\end{aligned}
$$

where the inequalities are straightforward. Substituting (3.15) into (3.6) with $\mu=0$ yields

$$
\frac{\mathrm{d}}{\mathrm{d} t}\left(\|\omega\|^{2}+\|j\|^{2}\right)+2 \nu\|\nabla \omega\|^{2} \leqslant 2 \frac{\|j\|_{\infty}}{\|j\|}\|\omega\|\left(\|\omega\|^{2}+\|j\|^{2}\right) .
$$

Similar to the derivation of (3.9), we apply Gronwall's inequality to (3.16) or directly integrate it with the integrating factor $\exp \left\{-2 \int_{0}^{t}\|j\|_{\infty}\|\omega\| /\|j\| \mathrm{d} \tau\right\}$ and obtain

$$
\|\omega\|^{2}+\|j\|^{2}+2 v \int_{0}^{t}\|\nabla \omega\|^{2} \mathrm{~d} \tau \leqslant\left(\left\|\omega_{0}\right\|^{2}+\left\|j_{0}\right\|^{2}\right) \exp \left\{2 \int_{0}^{t} \frac{\|j\|_{\infty}}{\|j\|}\|\omega\| \mathrm{d} \tau\right\} .
$$

The right-hand side of (3.17) would remain bounded as long as the ratio $\|j\|_{\infty} /\|j\|$ does not grow too rapidly with time, that is when $j$ does not exhibit extreme 
concentration. More specifically, if $\|j\|_{\infty} /\|j\| \leqslant F(t)$ where $F(t)$ satisfies $\int_{0}^{t} F(\tau)^{2} \mathrm{~d} \tau<$ $\infty$, then by Hölder's inequality and (3.14) we have

$$
\begin{aligned}
\int_{0}^{t} \frac{\|j\|_{\infty}}{\|j\|}\|\omega\| \mathrm{d} \tau & \leqslant \int_{0}^{t} F(\tau)\|\omega\| \mathrm{d} \tau \\
& \leqslant\left[\int_{0}^{t} F(\tau)^{2} \mathrm{~d} \tau\right]^{1 / 2}\left[\int_{0}^{t}\|\omega\|^{2} \mathrm{~d} \tau\right]^{1 / 2}<\infty .
\end{aligned}
$$

It follows that

$$
\|\omega\|^{2}+\|j\|^{2}+2 v \int_{0}^{t}\|\nabla \omega\|^{2} \mathrm{~d} \tau<\infty
$$

which implies both

$$
\int_{0}^{t}\|\omega\|_{B M O} \mathrm{~d} \tau \leqslant C \int_{0}^{t}\|\nabla \omega\| \mathrm{d} \tau<\infty
$$

and

$$
\begin{aligned}
\int_{0}^{t}\|j\|_{B M O} \mathrm{~d} \tau & \leqslant 2 \int_{0}^{t}\|j\|_{\infty} \mathrm{d} \tau \leqslant 2 \int_{0}^{t} F(\tau)\|j\| \mathrm{d} \tau \\
& \leqslant 2\left[\int_{0}^{t} F(\tau)^{2} \mathrm{~d} \tau\right]^{1 / 2}\left[\int_{0}^{t}\|j\|^{2} \mathrm{~d} \tau\right]^{1 / 2}<\infty .
\end{aligned}
$$

Hence, (2.5) holds and regularity follows.

Alternatively, consider the estimate

$$
\int j B(\nabla \boldsymbol{u}, \nabla \boldsymbol{b}) \mathrm{d} \boldsymbol{x} \leqslant\|j\|\|\nabla \boldsymbol{u}\|_{\infty}\|\nabla \boldsymbol{b}\|=\|\nabla \boldsymbol{u}\|_{\infty}\|j\|^{2} .
$$

Substituting (3.22) into (3.6) with $\mu=0$ yields

$$
\frac{\mathrm{d}}{\mathrm{d} t}\left(\|\omega\|^{2}+\|j\|^{2}\right)+2 v\|\nabla \omega\|^{2} \leqslant 2\|\nabla \boldsymbol{u}\|_{\infty}\|j\|^{2} .
$$

Now let $F(t)$ be a function such that

$$
\frac{\|\nabla \boldsymbol{u}\|_{\infty}}{\|\omega\|} \leqslant F(t)
$$

By substituting this into (3.23) we obtain

$$
\frac{\mathrm{d}}{\mathrm{d} t}\left(\|\omega\|^{2}+\|j\|^{2}\right)+2 v\|\nabla \omega\|^{2} \leqslant 2 F(t)\|\omega\|\left(\|\omega\|^{2}+\|j\|^{2}\right),
$$

which, upon integration, yields

$$
\|\omega\|^{2}+\|j\|^{2}+2 v \int_{0}^{t}\|\nabla \omega\|^{2} \mathrm{~d} \tau \leqslant\left(\left\|\omega_{0}\right\|^{2}+\left\|j_{0}\right\|^{2}\right) \exp \left\{2 \int_{0}^{t} F(\tau)\|\omega\| \mathrm{d} \tau\right\} .
$$

Suppose $\int_{0}^{t} F(\tau)^{2} \mathrm{~d} \tau<\infty$. Then the exponential factor on the right-hand side of (3.26) is bounded since by Hölder's inequality and (3.14) we have

$$
\int_{0}^{t} F(\tau)\|\omega\| \mathrm{d} \tau \leqslant\left[\int_{0}^{t} F(\tau)^{2} \mathrm{~d} \tau\right]^{1 / 2}\left[\int_{0}^{t}\|\omega\|^{2} \mathrm{~d} \tau\right]^{1 / 2}<\infty .
$$

So the left-hand side of (3.26) is bounded and we recover (3.19). 
We now establish the boundedness of $\|\nabla \omega\|^{2}+\|\nabla j\|^{2}$. Such a result immediately implies the improved CKS criterion (2.5) thanks to (A 8) given in Appendix. By multiplying (3.3) and (3.4) by $\Delta \omega$ and $\Delta j$, respectively, summing up and integrating the resulting equation we obtain

$$
\begin{aligned}
\frac{1}{2} \frac{\mathrm{d}}{\mathrm{d} t}\left(\|\nabla \omega\|^{2}+\|\nabla j\|^{2}\right)= & \int T_{1}(\nabla \boldsymbol{u}, \nabla \omega, \nabla \omega) \mathrm{d} \boldsymbol{x}+\int T_{2}(\nabla \boldsymbol{u}, \nabla j, \nabla \nabla \boldsymbol{b}) \mathrm{d} \boldsymbol{x} \\
& +\int T_{3}(\nabla \boldsymbol{b}, \nabla \nabla \boldsymbol{u}, \nabla j) \mathrm{d} \boldsymbol{x}-v\|\Delta \omega\|^{2} .
\end{aligned}
$$

In (3.28), the trilinear terms

$$
\begin{aligned}
& T_{1}=-\sum_{m, n=1}^{2}\left(\partial_{m} u_{n}\right)\left(\partial_{n} \omega\right)\left(\partial_{m} \omega\right) \\
& T_{2}=-\sum_{m, n=1}^{2}\left(\partial_{m} u_{n}\right)\left(\partial_{n} j\right)\left(\partial_{m} j\right)+\sum_{m=1}^{2} B\left(\nabla \boldsymbol{u}, \nabla \partial_{m} \boldsymbol{b}\right)\left(\partial_{m} j\right) \\
& T_{3}=\sum_{m, n=1}^{2}\left(\partial_{m} b_{n}\right)\left[\left(\partial_{n} j\right)\left(\partial_{m} \omega\right)+\left(\partial_{n} \omega\right)\left(\partial_{m} j\right)\right]+\sum_{m=1}^{2} B\left(\nabla \partial_{m} \boldsymbol{u}, \nabla \boldsymbol{b}\right)\left(\partial_{m} j\right)
\end{aligned}
$$

involve derivatives of $\boldsymbol{u}$ and $\boldsymbol{b}$ up to second-order only (i.e. up to first-order derivatives of $\omega$ and $j$ ). Here $\partial_{1}=\partial / \partial x, \partial_{2}=\partial / \partial y$ and $B(\cdot, \cdot)$ is the bilinear form defined in (3.5). These desired forms of $T_{i}$ have been obtained by integration by parts, which effectively redistributes the derivatives among $\boldsymbol{u}, \boldsymbol{b}, \omega$ and $j$, together with making use of the divergence-free properties $\boldsymbol{\nabla} \cdot \boldsymbol{u}=\boldsymbol{\nabla} \cdot \boldsymbol{b}=0$. It is clear that

$$
\left|T_{1}\right| \leqslant c|\nabla \boldsymbol{u}||\nabla \omega|^{2}, \quad\left|T_{2}\right| \leqslant c|\nabla \boldsymbol{u}||\nabla j||\nabla \nabla \boldsymbol{b}|, \quad\left|T_{3}\right| \leqslant c|\nabla \boldsymbol{b}||\nabla \nabla \boldsymbol{u}||\nabla j| .
$$

Now the triple-product terms $\int T_{i} \mathrm{~d} \boldsymbol{x}$ in (3.28) can be estimated in a straightforward manner. First, we have

$$
\left|\int T_{1} \mathrm{~d} \boldsymbol{x}\right| \leqslant c \int\left|\nabla \boldsymbol{u}\left\|\left.\nabla \omega\right|^{2} \mathrm{~d} \boldsymbol{x} \leqslant c\right\| \nabla \boldsymbol{u}\left\|_{\infty}\right\| \nabla \omega\left\|^{2} \leqslant c F(t)\right\| \omega\|\| \nabla \omega \|^{2} .\right.
$$

Second

$$
\left|\int T_{2} \mathrm{~d} \boldsymbol{x}\right| \leqslant c \int\left|\nabla \boldsymbol{u}\|\nabla j|| \nabla \nabla \boldsymbol{b} \mid \mathrm{d} \boldsymbol{x} \leqslant c\| \nabla \boldsymbol{u}\left\|_{\infty}\right\| \nabla j\left\|^{2} \leqslant c F(t)\right\| \omega\|\| \nabla j \|^{2} .\right.
$$

Third

$$
\begin{aligned}
\left|\int T_{3} \mathrm{~d} \boldsymbol{x}\right| & \leqslant c \int|\nabla \boldsymbol{b}\|\nabla \nabla \boldsymbol{u}\| \nabla j| \mathrm{d} \boldsymbol{x} \\
& \leqslant c\|\nabla \boldsymbol{b}\|_{L^{4}}\|\nabla \nabla \boldsymbol{u}\|_{L^{4}}\|\nabla j\| \leqslant c\|j\|_{L^{4}}\|\nabla \omega\|_{L^{4}}\|\nabla j\| \\
& \leqslant c\|j\|^{1 / 2}\|\nabla \omega\|^{1 / 2}\|\nabla j\|^{3 / 2}\|\Delta \omega\|^{1 / 2} \\
& \leqslant C\|j\|^{2 / 3}\|\nabla \omega\|^{2 / 3}\|\nabla j\|^{2}+v\|\Delta \omega\|^{2},
\end{aligned}
$$

where Gagliardo-Nirenberg's and Young's inequalities have been used in the penultimate and final steps, respectively. By substituting the above estimates for the triple-product terms into (3.28) we obtain

$$
\frac{\mathrm{d}}{\mathrm{d} t}\left(\|\nabla \omega\|^{2}+\|\nabla j\|^{2}\right) \leqslant C\left(F(t)\|\omega\|+\|j\|^{2 / 3}\|\nabla \omega\|^{2 / 3}\right)\left(\|\nabla \omega\|^{2}+\|\nabla j\|^{2}\right) .
$$


Integrating (3.36) yields

$$
\begin{aligned}
\|\nabla \omega\|^{2}+\|\nabla j\|^{2} \leqslant & \left(\left\|\nabla \omega_{0}\right\|^{2}+\left\|\nabla j_{0}\right\|^{2}\right) \\
& \times \exp \left\{C \int_{0}^{t}\left(F(\tau)\|\omega\|+\|j\|^{2 / 3}\|\nabla \omega\|^{2 / 3}\right) \mathrm{d} \tau\right\} .
\end{aligned}
$$

Thanks to the assumption on $F(t)$ and (3.19), we have

$$
\begin{aligned}
\int_{0}^{t}\left(F(\tau)\|\omega\|+\|j\|^{2 / 3}\|\nabla \omega\|^{2 / 3}\right) \mathrm{d} \tau \leqslant & \left(\int_{0}^{t} F(\tau)^{2} \mathrm{~d} \tau\right)^{1 / 2}\left(\int_{0}^{t}\|\omega\|^{2} \mathrm{~d} \tau\right)^{1 / 2} \\
& +\left(\int_{0}^{t}\|j\| \mathrm{d} \tau\right)^{2 / 3}\left(\int_{0}^{t}\|\nabla \omega\|^{2} \mathrm{~d} \tau\right)^{1 / 3}<\infty .
\end{aligned}
$$

So $\|\nabla \omega\|^{2}+\|\nabla j\|^{2}$ remains bounded. Hence, the improved CKS criterion holds and regularity follows.

In conclusion, we see that for solution regularity beyond $t=T$ of the 2D MHD system with $P m=\infty$, it is sufficient to require either

$$
\frac{\|j\|_{\infty}}{\|j\|} \leqslant C(T-t)^{-\alpha} \quad \text { or } \quad \frac{\|\nabla u\|_{\infty}}{\|\omega\|} \leqslant C(T-t)^{-\alpha}
$$

for $\alpha<1 / 2$.

In passing, it is worth mentioning that for ideal dynamics, as well as for all $\mathrm{Pm}$, one has the following regularity criterion

$$
\int_{0}^{T}\|\nabla j\|_{\infty} \mathrm{d} t<\infty
$$

Indeed, given (3.40) and energy conservation, the finiteness of $\int_{0}^{T}\|j\|_{\infty} \mathrm{d} t$ follows by applying the Gagliardo-Nirenberg inequality. Moreover, the vorticity equation (3.3) immediately implies that $\|\omega\|_{\infty}$ is bounded over $[0, T]$. Hence, both $\|j\|_{\infty}$ and $\|\omega\|_{\infty}$ are integrable over $[0, T]$. Thus, (2.4) holds, and regularity follows.

\section{Numerical results}

This section presents the results from a series of numerical simulations, which support the above theories and confirm the mathematical result of the linear scaling of $v\|\omega\|^{2}$ with $v$ in the limit $P m \rightarrow 0$. Interestingly, a slightly less rapid decrease of $\mu\|j\|^{2}$ with $\mu$ is observed in the large $P m$ regime.

Equations (2.1) and (2.2), rewritten in the form

$$
\begin{aligned}
\frac{\partial \omega}{\partial t}+J(\psi, \omega) & =J(a, \Delta a)+v \Delta \omega, \\
\frac{\partial a}{\partial t}+J(\psi, a) & =\mu \Delta a,
\end{aligned}
$$

where $\psi$ is the streamfunction, $a$ is the magnetic potential and $J(\cdot, \cdot)$ denotes the Jacobian, were integrated numerically using a pseudo-spectral method in a periodic domain of side $2 \pi$. A fourth-order Runge-Kutta time-stepping procedure was used, with the dissipative terms incorporated exactly using integrating factors. The initial magnetic modes lay within the wavenumber range [5,8], having random phases and 

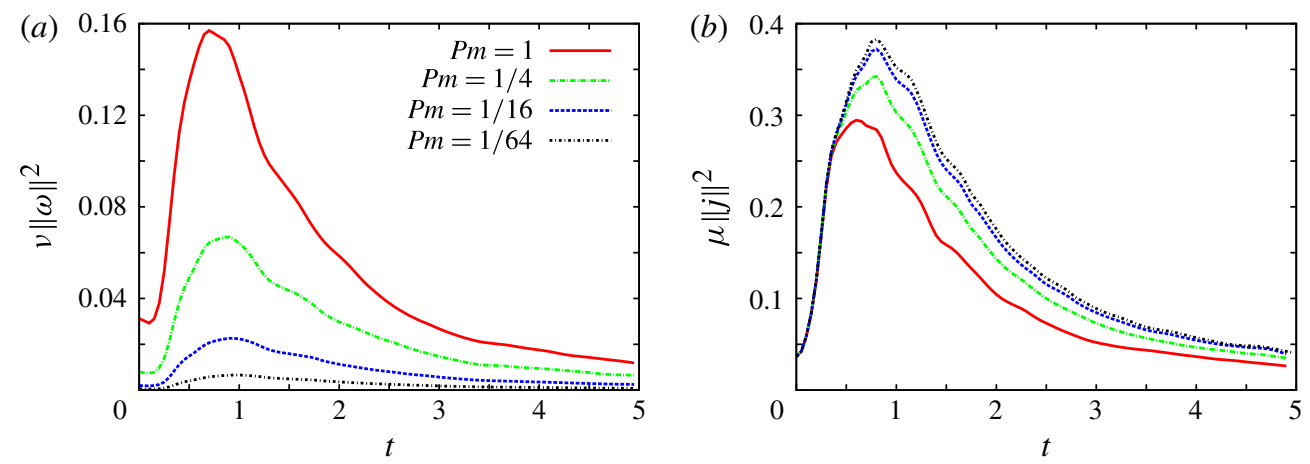

FIgURE 1. (Colour online) Kinetic $(a)$ and magnetic $(b)$ energy dissipation rates versus time for $P m=1,1 / 4,1 / 16,1 / 64$.

an energy of 0.5 . The initial mechanical modes were also confined to the range $[5,8]$, having random phases and an energy of 0.5 . This amounts to a total energy of 1.0. Seven different values of $P m$ were chosen: $P m=1 / 64,1 / 16,1 / 4,1,4,16,64$. The case $P m=1$ corresponds to $v=\mu=8 \times 10^{-4}$. For $P m \neq 1$, we fixed one diffusion coefficient at $8 \times 10^{-4}$ and decreased the other accordingly. The cases $P m=1 / 64$ and $P m=64$ were simulated at the highest resolution of $8192 \times 8192$. For $P m=1 / 64$, the magnetic and kinetic Reynolds numbers $R m$ and $R e$, defined in terms of the maximum energy dissipation rates (see Blackbourn \& Tran 2012; Tran \& Blackbourn 2012; Tran $\& \mathrm{Yu} 2012$ ) are $R m=10500$ and $R e=173700$. On the other hand, for $P m=64$, these numbers are $R m=325000$ and $R e=9700$. For each $P m$, the energy conversion (primarily from kinetic to magnetic) was observed to become saturated when $t \gtrsim 1$, shortly after which the energy dissipation achieves its peak. In agreement with the findings of Tran \& Blackbourn (2012), the saturated ratio of magnetic to kinetic energy (not shown) have been found to be within the range [2,3].

Figure 1 shows the kinetic $(a)$ and magnetic $(b)$ energy dissipation rates versus time for $P m=1,1 / 4,1 / 16,1 / 64$. It can be seen that energy loss is primarily due to Ohmic dissipation, in agreement with previous studies for the regime of moderate and small Pm (cf. Brandenburg 2011a). The decrease of $v\|\omega\|^{2}$ is not quite linear with $P m$, but nonetheless appears to accelerate as $P m$ is decreased. This implies that the simulations have approached the regime of maximal enstrophy (more details below). The total energy dissipation rate versus time is shown in figure 2. As $P m$ is decreased, the time taken for each kinetic, magnetic and total energy dissipation rate to achieve its maximum increases, a result consistent with that of Blackbourn \& Tran (2012) for the case $P m=1$ when $v=\mu$ is decreased. Another feature in agreement with the results of Blackbourn \& Tran (2012) is the decrease of the peak of the total energy dissipation rate as $P m$ is decreased. Note, however, that for the present case, this peak decreases toward a positive limit (which is the maximum of $\mu\|j\|^{2}$ ) because $\mu$ is fixed. The vanishing of kinetic energy dissipation in the limit $P m \rightarrow 0$ partly justifies the numerical approach of Dritschel \& Tobias (2012), who simulated 2D MHD turbulence at low Pm using a conservative numerical scheme for the vorticity. Given strong support for solution regularity discussed below, this justification could be considered complete.

The evolution of the kinetic, magnetic and total energy dissipation rates for $P m=1,4,16,64$ are shown in figures 3 and 4. It can be seen that Ohmic dissipation 


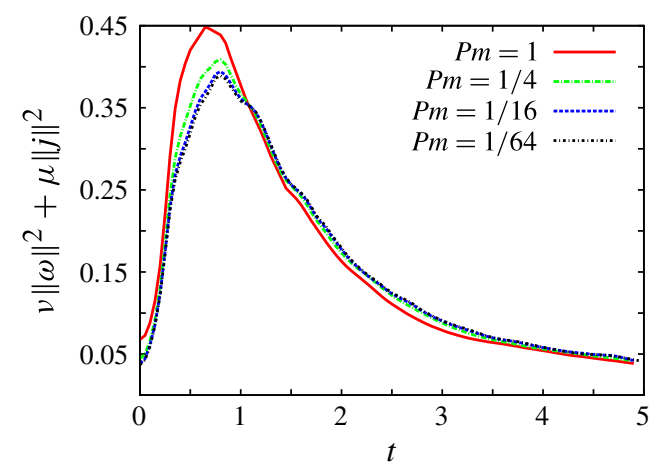

FIgURE 2. (Colour online) Total energy dissipation rates versus time for $P m=1,1 / 4,1 / 16,1 / 64$.

(a)

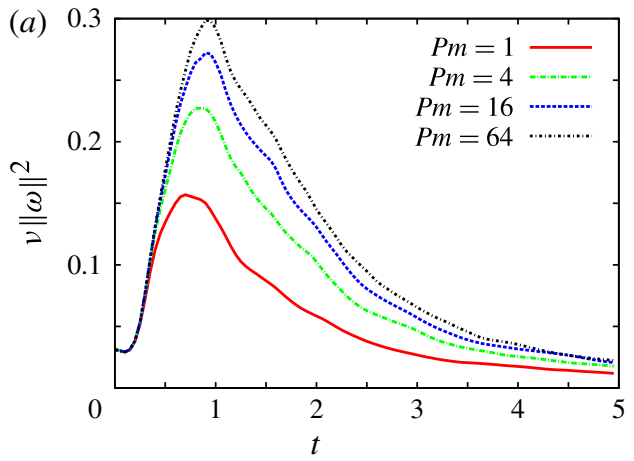

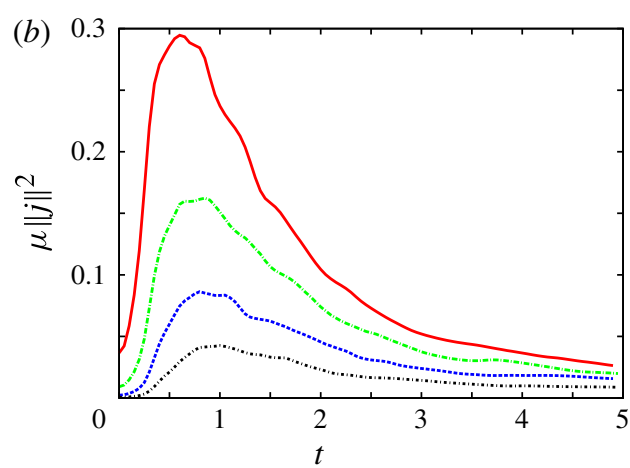

FIgURE 3. (Colour online) Kinetic $(a)$ and magnetic $(b)$ energy dissipation rates versus time for $P m=1,4,16,64$.

is greater than its viscous counterpart for the case $P m=1$ only. The latter overtakes the former for $P m=4$ and becomes dominant for $P m>4$. This confirms the earlier suggestion by Blackbourn \& Tran (2012) that Ohmic dissipation is not necessarily predominant for all $P m$. Like the cases $P m \leqslant 1$, the time of each maximum dissipation rate increases slowly as $\mathrm{Pm}$ is increased. Furthermore, the peak of the total energy dissipation rate decreases (presumably toward a positive limit, which is the maximum of $\left.v\|\omega\|^{2}\right)$ as $P m$ is increased. An interesting behaviour of $\mu\|j\|^{2}$ is that it decreases quite rapidly as $P m$ is increased from unity. The decrease is less rapid (approximately like $P m^{-0.6}$, more detail is given below) than that of $v\|\omega\|^{2}$ in the previous cases $(P m \leqslant 1)$ when $P m$ is decreased from unity. This means that a much wider range of $P m$ is required to access the regime of maximal $\|j\|^{2}$ if $\|j\|^{2}$ is to remain bounded in the limit $P m \rightarrow \infty$ (a behaviour suggested by further numerical results on the issue of regularity, see below).

In passing, it is worth noting that for the range of $\mathrm{Pm}$ under consideration, the ratio of kinetic to magnetic energy dissipation rates has an apparent power-law behaviour. Figure 5 shows the ratio of the maximum of $v\|\omega\|^{2}$ to that of $\mu\|j\|^{2}$ versus $P m$. For small $P m$, this ratio, say $r_{d}$, is given by $r_{d} \approx 0.57 \mathrm{Pm}^{0.83}$, which is slightly sublinear in $P m$. This behaviour of $r_{d}$ implies a similar behaviour of $v\|\omega\|^{2}$ because $\mu\|j\|^{2}$ 


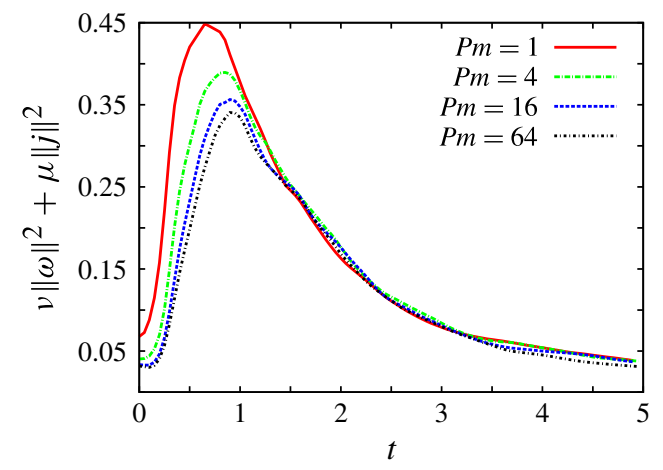

FIgURE 4. (Colour online) Total energy dissipation rates versus time for $P m=1,4,16,64$.

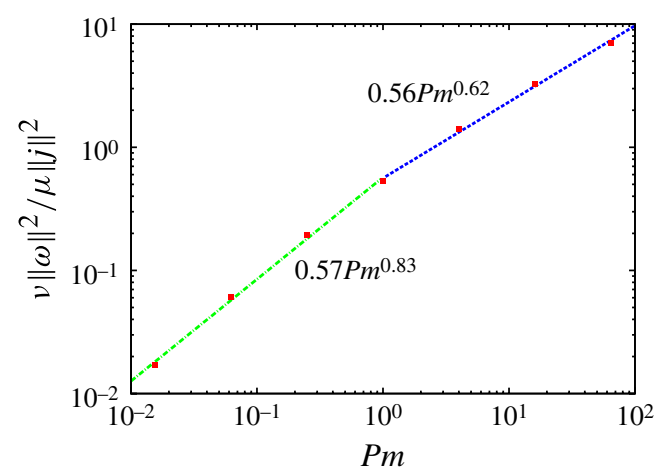

FIGURE 5. (Colour online) Ratio of peak kinetic to peak magnetic energy dissipation rates versus magnetic Prandtl number. The straight lines are best fit lines.

becomes independent of $P m$ in the small $P m$ limit. The exponent 0.83 provides a quantitative measure of how close our simulations were to the maximal enstrophy regime. On the other hand, for large $P m, r_{d}$ scales approximately as $0.56 P m^{0.62}$. The exponent 0.62 is significantly less than unity, implying that our simulations were still quite far from the expected regime of maximal $\|j\|$, if such a regime exists. Interestingly, power-law behaviour of a ratio similar to $r_{d}$ was observed in a previous study. For a mechanically forced 3D model, Brandenburg (2011b) found $r_{d} \approx 0.6 P m^{0.6}$ over six decades of $P m$, where his $r_{d}$ is the ratio of averaged energy dissipation rates. While the analysis in $\S 3$ has provided a mathematical proof that the exponent 0.83 in $r_{d} \approx 0.57 \mathrm{Pm}^{0.83}$ increases toward unity for lower ranges of $\mathrm{Pm}$, the exponent 0.6 in Brandenburg's result appears to be robust.

As far as we know, no algorithms for computing the BMO norm have been put forth in the literature. Given the unavailability of such algorithms, we monitored the (slightly stronger) ratio $\|\omega\|_{\infty} /\|\omega\|$ instead of $\|\omega\|_{B M O} /\|\omega\|$, although the boundedness of the latter is sufficient for regularity when $P m=0$. Figure $6(a)$ shows the evolution of $\|\omega\|_{\infty} /\|\omega\|$ for $P m=1,1 / 4,1 / 16,1 / 64$. As $P m$ is decreased by a factor of 64 , $\|\omega\|_{\infty} /\|\omega\|$ approximately increases by a factor of 2 . This can be considered strong support for the possibility $\|\omega\|_{\infty} /\|\omega\|<\infty$ in the limit $P m \rightarrow 0$. A quantitative dependence of $\|\omega\|_{\infty} /\|\omega\|$ on $P m$ is required to address this possibility and, hence, 

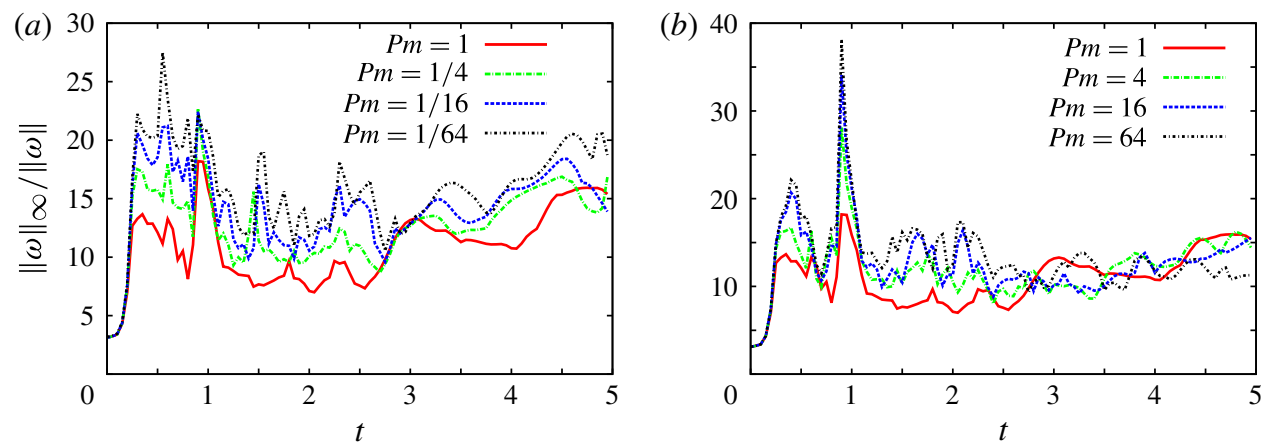

FIgure 6. (Colour online) Plots of $\|\omega\|_{\infty} /\|\omega\|$ versus time for $P m=1,1 / 4,1 / 16,1 / 64$ (a) and for $P m=1,4,16,64(b)$.

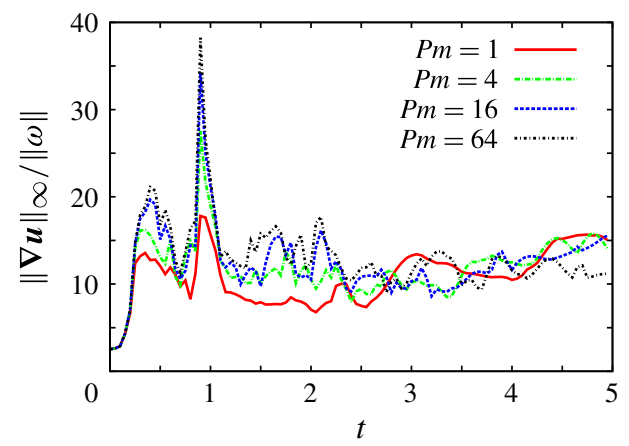

FIgURE 7. (Colour online) Plots of $\|\nabla \boldsymbol{u}\|_{\infty} /\|\omega\|$ versus time for $P m=1,4,16,64$.

the issue of regularity for $P m=0$, but is not possible by the present set of simulations without undermining the Reynolds numbers. For comparison, we have included figure $6(b)$, which describes the evolution of $\|\omega\|_{\infty} /\|\omega\|$ for the cases $P m=1,4,16,64$. It will be seen shortly that $\|\omega\|_{\infty} /\|\omega\|$ and $\|\nabla \boldsymbol{u}\|_{\infty} /\|\omega\|$ are virtually the same for these cases.

With regard to the issue of regularity for the case $P m=\infty$, we have obtained evidence as strong as figure $6(a)$ for the case $P m=0$. Figure 7 describes the evolution of the ratio $\|\nabla \boldsymbol{u}\|_{\infty} /\|\omega\|$ for $P m=1,4,16,64$. As $P m$ is increased from unity to 64, this ratio approximately increases twofold, thereby suggesting the possibility $\|\nabla \boldsymbol{u}\|_{\infty} /\|\omega\|<\infty$ in the limit $P m \rightarrow \infty$. Note that no appreciable discrepancies between $\|\nabla \boldsymbol{u}\|_{\infty} /\|\omega\|$ and $\|\omega\|_{\infty} /\|\omega\|$ can be observed (cf. figure 6). This makes sense since $\|\nabla \boldsymbol{u}\|_{\infty}$ and $\|\omega\|_{\infty}$ are not expected to differ by much, although the fields $|\omega|$ and $|\nabla \boldsymbol{u}|$ may disagree substantially on local basis. Interestingly, vortex and velocity gradient 'filaments' look alike (see below), suggesting that $|\nabla \boldsymbol{u}| \approx|\omega|$ at large values.

Figure $8(b)$ shows the plots of $\|j\|_{\infty} /\|j\|$ versus time for $P m=1,4,16,64$. It can be seen that over the range $P m \in[1,64],\|j\|_{\infty} /\|j\|$ increases approximately threefold. This provides slightly weaker support for regularity than the milder behaviour of $\|\nabla \boldsymbol{u}\|_{\infty} /\|\omega\|$ (with twofold increase) discussed above. For comparison, figure 8(a) 

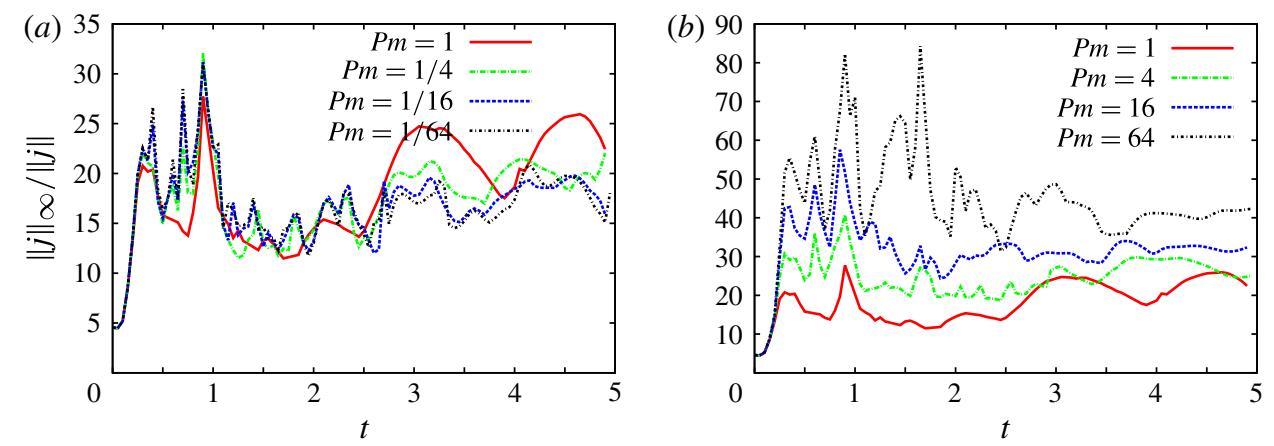

FIgURE 8. (Colour online) Plots of $\|j\|_{\infty} /\|j\|$ versus time for $P m=1,1 / 4,1 / 16,1 / 64$ (a) and for $P m=1,4,16,64(b)$.

(a)

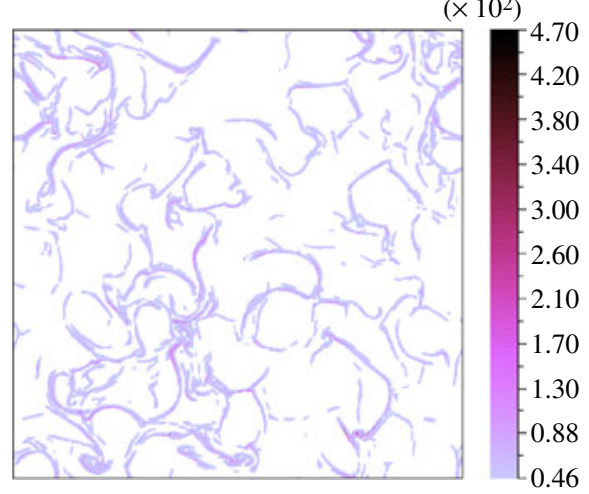

(b)

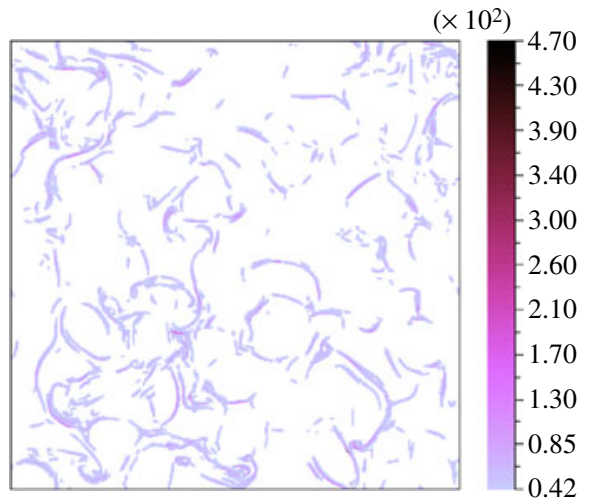

FIgURE 9. (Colour online) Vorticity $(a)$ and current $(b)$ fields at $t=1$ for $P m=1 / 64$. The images are for $|\omega| \geqslant 2\|\omega\| \approx 4.6 \times 10^{1}$ and $|j| \geqslant 2\|j\| \approx 4.2 \times 10^{1}$.

shows a relatively much weaker response of $\|j\|_{\infty} /\|j\|$ to the decrease of $P m$ from unity to $1 / 64$.

The distributions of large values of the fields $|\omega|,|j|$ and $|\nabla \boldsymbol{u}|$ are of interest as these distributions provide a sense of the magnitudes of the ratios discussed above. Figures 9-11 show the images of $|\omega|$ for $|\omega| \geqslant 2\|\omega\|,|j|$ for $|j| \geqslant 2\|j\|$ and $|\nabla \boldsymbol{u}|$ for $|\nabla \boldsymbol{u}| \geqslant 2\|\omega\|$, for the cases $P m=1 / 64$ and $P m=64$ at $t=1.0$. This time is shortly after the energy dissipation in each case becomes greatest. For $P m=1 / 64$, the vortex and current filamentary structures are comparable in magnitude and density. On the other hand, for $P m=64$, these 'filaments' are significantly less dense and are an order of magnitude greater than their $P m=1 / 64$ counterparts. In any case, at both magnetic Prandtl numbers vortex filaments are qualitatively the same (this is true for velocity gradient filaments, whose image for the case $P m=1 / 64$ is omitted). So the change in $|\omega|$ (and $|\nabla \boldsymbol{u}|$ ) is relatively mild over a significantly wide range of $P m$. The heavy population of vortex and velocity gradient filaments is indicative of moderate values of $\|\omega\|_{\infty} /\|\omega\|$ and $\|\nabla \boldsymbol{u}\|_{\infty} /\|\omega\|$. On the other hand, over the same range of $P m,|j|$ can be seen to change significantly. Nonetheless, this poses no risk to the possibility of regularity, given the mild behaviour of $\|j\|_{\infty} /\|j\|$ (and of $\|\nabla \boldsymbol{u}\|_{\infty} /\|\omega\|$ ). An interesting 
(a)

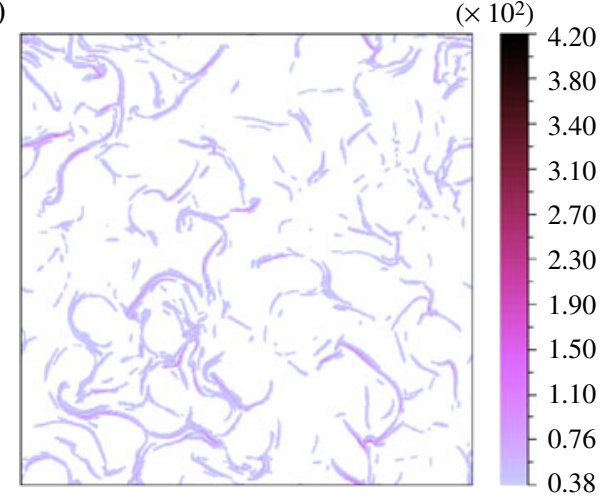

(b)

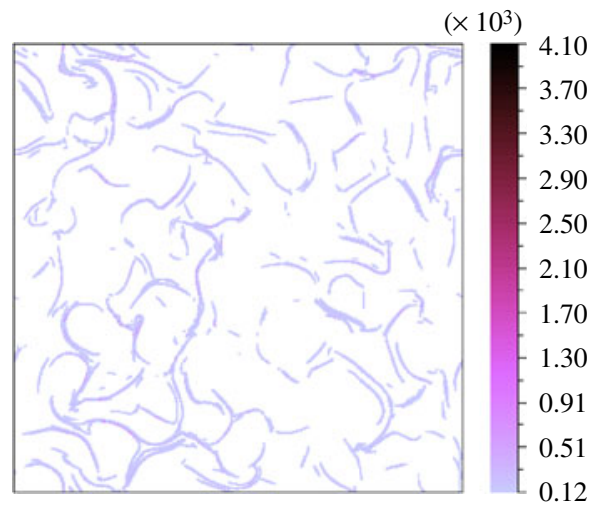

FIgURE 10. (Colour online) Vorticity $(a)$ and current $(b)$ fields at $t=1$ for $P m=64$. The images are for $|\omega| \geqslant 2\|\omega\| \approx 3.8 \times 10^{1}$ and $|j| \geqslant 2\|j\| \approx 1.2 \times 10^{2}$.

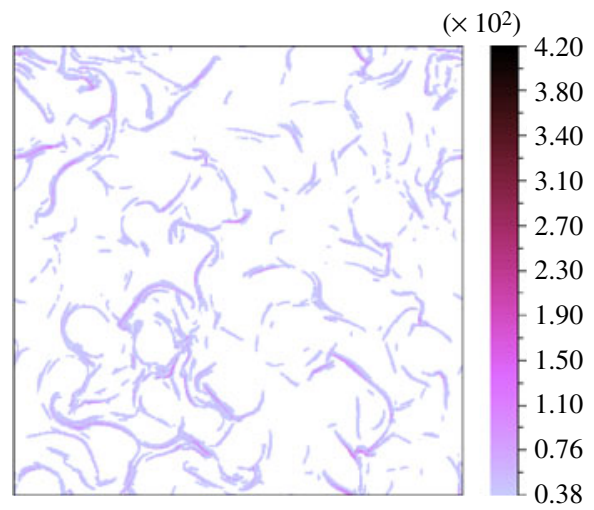

FIgURE 11. (Colour online) Velocity gradient field at $t=1$ for $P m=64$. The image is for $|\nabla \boldsymbol{u}| \geqslant 2\|\omega\| \approx 3.8 \times 10^{1}$.

feature to note is that the images of $|\omega|$ and $|\nabla \boldsymbol{u}|$ for $P m=64$ differ only in minute details.

At $t=3$, the turbulence can be considered fully developed. Figures 12-14 are similar to figures 9-11, albeit at $t=3$, and are presented here for comparison. Somewhat surprisingly, over the time interval [1,3], $\|\omega\|_{\infty},\|\nabla \boldsymbol{u}\|_{\infty}$ and $\|j\|_{\infty}$ decrease much more rapidly than their $L^{2}$ norm counterparts as the scales of the figures indicate (see also figures 6-8). This is in a sharp contrast to 2D Navier-Stokes turbulence, for which $\|\omega\|$ decays much more rapidly than $\|\omega\|_{\infty}$ throughout the course of evolution (Dritschel et al. 2007). While it is fairly easy to understand why $\|\omega\|_{\infty}$ is better conserved than $\|\omega\|$ in 2D Navier-Stokes turbulence, we have no obvious explanation for the above observation in the present case.

\section{Concluding remarks}

We have studied both theoretically and numerically 2D MHD turbulence in the limits of infinite and vanishing magnetic Prandtl number $P m=v / \mu$, as well as the 
(a)

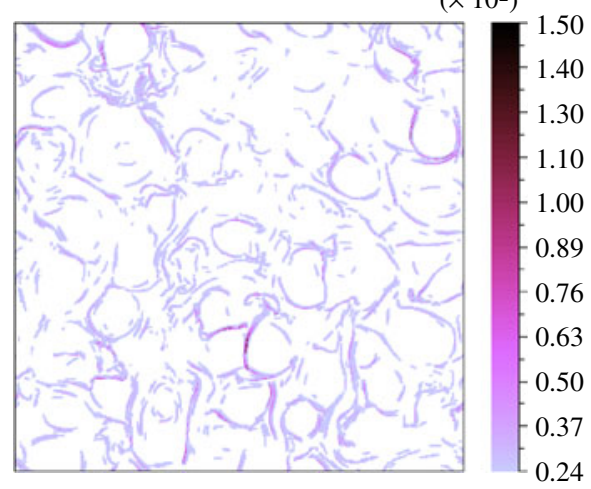

(b)

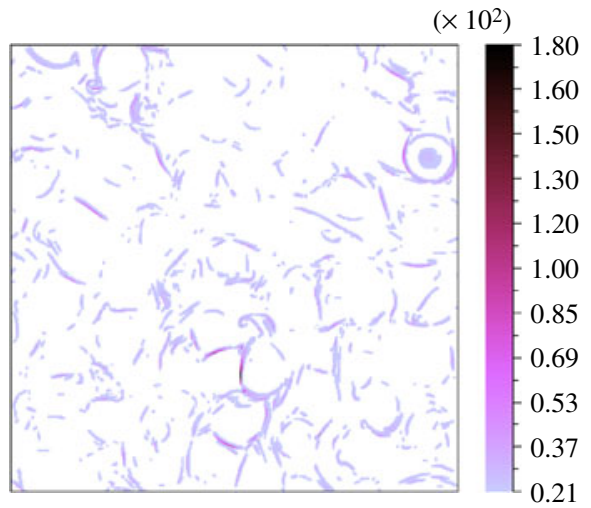

FIGURE 12. (Colour online) Vorticity $(a)$ and current $(b)$ fields at $t=3$ for $P m=1 / 64$. The images are for $|\omega| \geqslant 2\|\omega\| \approx 2.4 \times 10^{1}$ and $|j| \geqslant 2\|j\| \approx 2.1 \times 10^{1}$.

(a)

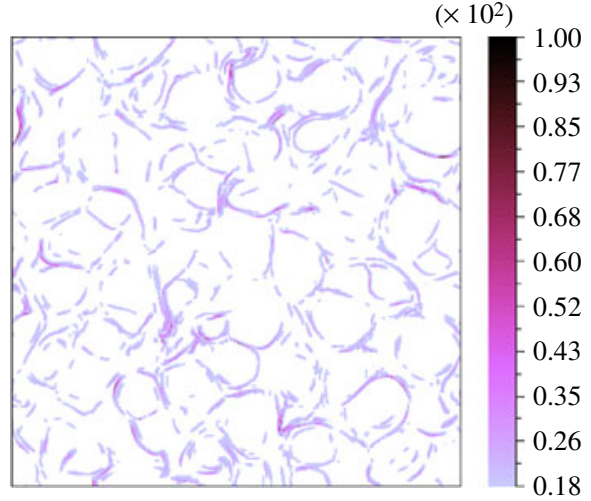

(b)

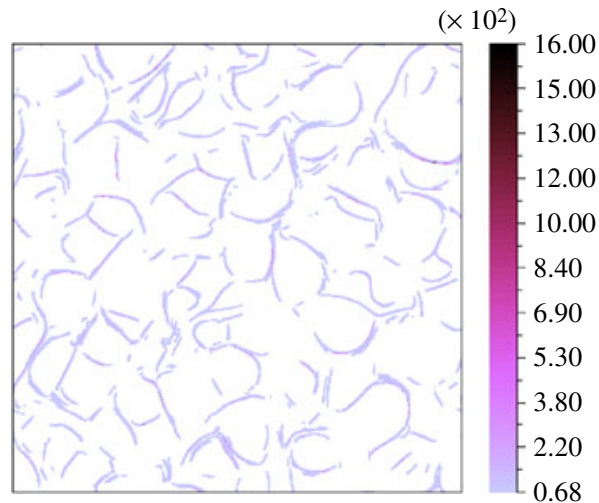

FIGURE 13. (Colour online) Vorticity $(a)$ and current $(b)$ fields at $t=3$ for $P m=64$. The images are for $|\omega| \geqslant 2\|\omega\| \approx 1.8 \times 10^{1}$ and $|j| \geqslant 2\|j\| \approx 6.8 \times 10^{1}$.

partial dissipation cases, i.e. $P m=0$ and $P m=\infty$. For positive magnetic diffusivity $\mu>0,\|\nabla j\|^{2}$ is integrable over $[0, \infty)$. Furthermore, both $\|\omega\|^{2}$ and $\|j\|^{2}$ are uniformly bounded independently of the viscosity $v$. This means that given $v \rightarrow 0$ at fixed $\mu>0$, the kinetic energy dissipation rate $v\|\omega\|^{2}$ tends to zero. For sufficiently small $P m$, the approach $v\|\omega\|^{2} \rightarrow 0$ is linear in $v$. Regularity criteria have been derived for the partial dissipation cases. When $P m=0$, smooth solutions remain smooth up to $t=T$ provided that $\|\omega\|_{B M O} /\|\omega\| \leqslant C(T-t)^{-\alpha}$, for $\alpha<1$. When $P m=\infty$, either $\|j\|_{\infty} /\|j\| \leqslant C(T-t)^{-\alpha}$ or $\|\nabla \boldsymbol{u}\|_{\infty} /\|\omega\| \leqslant C(T-t)^{-\alpha}$, for $\alpha<1 / 2$, is sufficient. The latter is physically plausible given the presence of viscous effects. The results from high-resolution numerical simulations over the range $P m \in[1 / 64,64]$ support these criteria and demonstrate the nearly linear behaviour of the kinetic energy dissipation rate with $v$ for fixed $\mu>0$. As $P m$ is decreased from unity, the ratio $\|\omega\|_{\infty} /\|\omega\|$ has been observed to increase relatively slowly. On the other hand, an equally slow increase of $\|\nabla \boldsymbol{u}\|_{\infty} /\|\omega\|$ has been observed when $P m$ is increased from unity. These results lend strong support for solution regularity for the partial dissipation cases. 


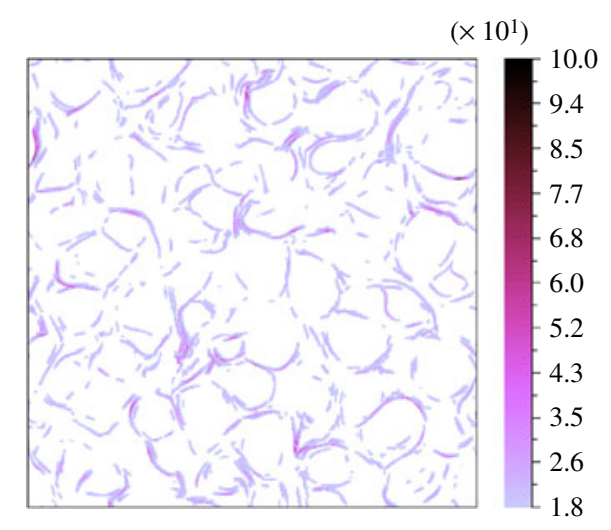

FIgURE 14. (Colour online) Velocity gradient field at $t=3$ for $P m=64$. The image is for $|\nabla \boldsymbol{u}| \geqslant 2\|\omega\| \approx 1.8 \times 10^{1}$.

Further evidence for the mild behaviour of the said ratios has been observed from the filamentary structures of large values of the vorticity and velocity gradient fields. These filaments are heavily populated, an indication of moderate values of $\|\omega\|_{\infty} /\|\omega\|$ and $\|\nabla \boldsymbol{u}\|_{\infty} /\|\omega\|$.

\section{Acknowledgements}

We thank the anonymous referees for helpful comments. L.A.K.B. was supported by an EPSRC post-graduate studentship. X.Y. was supported by an NSERC discovery grant and a startup grant from the Faculty of Science of the University of Alberta.

\section{Appendix. The space $\mathrm{BMO}\left(R^{n}\right)$}

The space $\mathrm{BMO}\left(R^{n}\right)$ of BMO functions in $R^{n}$ plays an important role in the theory of partial differential equations. This appendix provides a brief review of this special space, together with some of its basic properties used in this study.

Let $f(\boldsymbol{x})$ be a locally integrable function in $R^{n}$ and $Q \subset R^{n}$ be an $n$-dimensional cube with measure (volume) $\mu(Q)$. The mean oscillation of $f(\boldsymbol{x})$ over $Q$ is defined by the quantity

$$
\frac{1}{\mu(Q)} \int_{Q}\left|f(\boldsymbol{x})-f_{Q}\right| \mathrm{d} \boldsymbol{x},
$$

where $f_{Q}$ stands for the average of $f$ over $Q$, i.e.

$$
f_{Q}:=\frac{1}{\mu(Q)} \int_{Q} f(\boldsymbol{x}) \mathrm{d} \boldsymbol{x} .
$$

The space $\operatorname{BMO}\left(R^{n}\right)$ is equipped with the norm

$$
\|f\|_{B M O}:=\sup _{\boldsymbol{x} \in R^{n}} \sup _{Q} \frac{1}{\mu(Q)} \int_{Q}\left|f(\boldsymbol{y})-f_{Q}\right| \mathrm{d} \boldsymbol{y}
$$

where the second supremum is taken over all cubes $Q$ containing $\boldsymbol{x}$. 
From this definition two things are immediately clear. First, the BMO norm is weaker than the $L^{\infty}$ norm. Indeed, one has

$$
\|f\|_{B M O} \leqslant 2\|f\|_{L^{\infty}} \text {. }
$$

Second, the BMO norm has the same scaling as that of its $L^{\infty}$ counterpart.

The BMO norm is slightly weaker than the $L^{\infty}$ norm, but not by much. More quantitatively, we have the following theorem.

TheOrem A.1 (Kozono \& Tanuichi 2000). Let $1<p<\infty$ and $s>n / p$. Given $f$ with $(-\Delta)^{s / 2} f \in L^{p}$, there exists a constant $C=C(n, p, s)$ such that

$$
\|f\|_{\infty} \leqslant C\left(1+\|f\|_{B M O}\left(1+\log ^{+}\left\|(-\Delta)^{s / 2} f\right\|_{L^{p}}\right)\right),
$$

where $\log ^{+} a=\log a$ if $a \geqslant 1$ and $\log ^{+} a=0$ if $0<a<1$.

This theorem, together with the relation $\|f\|_{B M O} \leqslant 2\|f\|_{L^{\infty}}$, means that the BMO and $L^{\infty}$ norms are almost equivalent, up to a logarithmic 'correction'. This fact enables Kozono \& Tanuichi (2000) to replace $\|\omega\|_{\infty}$ by $\|\omega\|_{B M O}$ in the BKM criterion, by essentially accommodating a logarithmic factor in the estimate of the growth rate of high-order derivatives of $\boldsymbol{u}$.

Some in-depth comparison between the BMO and $L^{\infty}$ spaces is possible on the basis of the following theorem.

THEOREM A.2 (John \& Nirenberg 1961). For any function $f(\boldsymbol{x}) \in$ BMO there are two positive constants $C_{1}$ and $C_{2}$ such that the following holds for any cube $Q$ and any $\lambda>0$.

$$
\mu\left(\left\{\boldsymbol{x} \in Q:\left|f(\boldsymbol{x})-f_{Q}\right|>\lambda\right\}\right) \leqslant C_{1} \mathrm{e}^{-C_{2} \lambda} \mu(Q) .
$$

Equation (A 6) means that 'extremal values' of a function in BMO are 'exponentially rare'. In comparison, such values of a function in $L^{\infty}$ have an abrupt 'cut-off' at a finite value. In fact, given $f(\boldsymbol{x}) \in L^{\infty}$, one has $\mu\left(\left\{\boldsymbol{x} \in Q:\left|f(\boldsymbol{x})-f_{Q}\right|>\right.\right.$ $\lambda\})=0$, for all $\lambda \geqslant 2\|f\|_{\infty}$. A typical example of a BMO function which is not in $L^{\infty}$ is $\ln |x|$.

In the context of partial differential equations, the space $\operatorname{BMO}\left(R^{n}\right)$ possesses many useful properties, most of which are not enjoyed by its $L^{\infty}$ cousin. In particular, the following two properties have been repeatedly employed in our analysis. These would fail when BMO is replaced by $L^{\infty}$.

(a) Singular integral operators are bounded from BMO to BMO. In particular,

$$
\|\nabla \boldsymbol{u}\|_{B M O} \leqslant C\|\omega\|_{B M O}, \quad\|\nabla \boldsymbol{b}\|_{B M O} \leqslant C\|j\|_{B M O} .
$$

(b) For any $f$ we have

$$
\|f\|_{B M O} \leqslant C\|\nabla f\|_{L^{n}}
$$

A more complete discussion of BMO spaces can be found in many texts on harmonic analysis, such as Stein (1993).

\section{REFERENCES}

Beale, J. T., Kato, T. \& MAJDA, A. 1984 Remarks on the breakdown of smooth solutions for the 3-D Euler equations. Commun. Math. Phys. 94, 61-64.

Beresnyak, A. 2011 Spectral slope and Kolmogorov constant of MHD turbulence. Phys. Rev. Lett.

106, 075001. 
Blackbourn, L. A. K. \& Tran, C. V. 2012 On energetics and inertial range scaling laws of two-dimensional magnetohydrodynamic turbulence. J. Fluid Mech. 703, 238-254.

Brandenburg, A. 2011a Nonlinear small-scale dynamos at low magnetic Prandtl numbers. Astrophys. J. 741, 92.

BRAnDEnburg, A. $2011 b$ Dissipation in dynamos at low and high magnetic Prandtl numbers. Astron. Nachr. 332, 51-56.

Caflisch, R. E., Klapper, I. \& Steel, G. 1997 Remarks on singularities, dimension, and energ dissipation for ideal hydrodynamics MHD. Commun. Math. Phys. 184, 443-455.

CAO, C. \& WU, J. 2011 Global regularity for the 2D MHD equations with mixed partial dissipation and magnetic diffusion. Adv. Math. 226, 1803-1822.

Chambers, K. \& Forbes, L. K. 2011 The magnetic Rayleigh-Taylor instability for inviscid and viscous fluids. Phys. Plasmas 18, 052101.

Dritschel, D. G. \& Tobias, S. M. 2012 Two-dimensional magnetohydrodynamic turbulence in the small magnetic Prandtl limit. J. Fluid Mech. 703, 85-98.

Dritschel, D. G., Tran, C. V. \& ScotT, R. K. 2007 Revisiting Batchelor's theory of two-dimensional turbulence. J. Fluid Mech. 591, 379-391.

Galtier, S., Pouquet, A. \& Mangeney, A. 2005 Spectral scaling laws for incompressible anisotropic magnetohydrodynamic turbulence. Phys. Plasmas 12, 092310.

Goldreich, P. \& SRIDHAR, S. 1995 Toward a theory of interstellar turbulence. II. Strong Alfvénic turbulence. Astrophys. J. 438, 763-775.

Iroshnikov, P. S. 1964 Turbulence of a conducting fluid in a strong magnetic field. Sov. Astron. 7, 566-571.

Iskakov, A. B., Schekochihin, A. A., Cowley, S. C., McWilliam, J. C. \& Proctor, M. R. E. 2007 Numerical demonstration of fluctuation dynamo at low magnetic Prandtl numbers. Phys. Rev. Lett. 98, 208501.

John, F. \& Nirenberg, L. 1961 On functions of bounded mean oscillation. Commun. Pure Appl. Maths 14, 415-426.

Kiselev, A., Nazarov, F. \& Volberg, A. 2007 Global well-posedness for the critical 2D dissipative quasi-geostrophic equation. Invent. Math. 167, 445-453.

Kozono, H. \& TANuichi, Y. 2000 Limiting case of the Sobolev inequality in BMO, with application to the Euler equations. Commun. Math. Phys. 214, 191-200.

Kraichnan, R. H. 1965 Inertial-range spectrum of hydromagnetic turbulence. Phys. Fluids 8, 1385-1387.

LEI, Z. \& ZHOU, Y. 2009 BKM's criterion and global weak solutions for magnetohydrodynamics with zero viscosity. Discrete Contin. Dyn. Syst. 25, 575-583.

MoffAtT, K. H. 1967 On the suppression of turbulence by a uniform magnetic field. J. Fluid Mech. 28, 571-592.

Ng, C. S., Bhattacharjee, A., Munsi, D., Isenberg, P. A. \& Smith, C. W. 2010 Kolmogorov versus Irosnikov-Kraichnan spectra: consequence for ion heating in the Solar wind. J. Geophys. Res. 115, A02101.

Nirenberg, L. 1959 On elliptic partial differential equations. Ann. Scuola Norm. Sup. Pisa 13, $115-162$.

OrszaG, S. A. \& TANG, C.-M. 1979 Small-scale structure of two-dimensional magnetohydrodynamic turbulence. J. Fluid Mech. 90, 129-143.

Pouquet, A. 1978 On two-dimensional magnetohydrodynamic turbulence. J. Fluid Mech. 88, 1-16.

Sridhar, S. \& Goldreich, P. 1994 Toward a theory of interstellar turbulence. I. Weak Alfvénic turbulence. Astrophys. J. 432, 612-621.

Stein, E. M. 1970 Singular Integrals and Differentiability Properties of Functions. Princeton University Press.

Stein, E. M. 1993 Harmonic Analysis: Real-variable Methods, Orthogonality, and Oscillatory Integrals. Princeton University Press.

Tobias, S. M. \& CAttaneo, F. 2008 Dynamo action in complex flows: the quick and the fast. J. Fluid Mech. 601, 101-122.

Tran, C. V. \& Blackbourn, L. A. K. 2012 A dynamical systems approach to fluid turbulence. Fluid Dyn. Res. 44, 031417. 
Tran, C. V., Blackbourn, L. A. K. \& Scott, R. K. 2011 Number of degrees of freedom and energy spectrum of surface quasi-geostrophic turbulence. J. Fluid Mech. 684, 427-440.

Tran, C. V. \& Dritschel, D. G. 2006 Vanishing enstrophy dissipation in two-dimensional Navier-Stokes turbulence in the inviscid limit. J. Fluid Mech. 559, 107-116.

TRAN, C. V. \& YU, X. 2012 Bounds for the number of degrees of freedom of magnetohydrodynamics turbulence in two and three dimensions. Phys. Rev. E 85, 066323.

Tran, C. V., YU, X. \& ZHAI, Z. $2013 a$ On global regularity of 2D generalized magnetohydrodynamic equations. J. Differential Equations 254, 4194-4216.

Tran, C. V., YU, X. \& ZHAI, Z. $2013 b$ Note on solution regularity of the generalized magnetohydrodynamic equations with partial dissipation. Nonlinear Anal. 85, 43-51.

Verma, M. K., Roberts, D. A., Goldstein, M. L., Gosh, S. \& Stribling, W. T. 1996 A numerical study of the nonlinear cascade of energy in magnetohydrodynamic turbulence. J. Geophys. Res. 101, 21619-21625.

WU, J. 2011 Global regularity for a class of generalized magnetohydrodynamic equations. J. Math. Fluid Mech. 13, 295-305. 